\title{
Positive solutions for a class of fractional infinite-point boundary value problems
}

Yongqing Wang ${ }^{1,2 *}$ (1) and Lishan Liü

${ }^{\text {*} C o r r e s p o n d e n c e: ~}$
wyqing9801@163.com
${ }^{1}$ School of Statistics, Qufu Normal
University, Qufu, P.R. China
${ }^{2}$ School of Mathematical Sciences,
Qufu Normal University, Qufu, P.R.
China
Full list of author information is
available at the end of the article

available at the end of the article

\begin{abstract}
In this paper, we consider a class of fractional differential equations with infinite-point boundary value conditions. Under some conditions concerning the spectral radius with respect to the relevant linear operator, both the existence of uniqueness and the nonexistence of positive solution are obtained by means of the iterative technique.
\end{abstract}

Keywords: Fractional differential equation; Positive solution; Infinite-point boundary value problem; Spectral radius

\section{Introduction}

In this paper, we consider the following fractional differential equations (FDE for short) with infinite-point boundary value conditions:

$$
\left\{\begin{array}{l}
D_{0+}^{\alpha} u(t)+a(t) f(t, u(t))=0, \quad 0<t<1, \\
u(0)=u^{\prime}(0)=\cdots=u^{(n-2)}(0)=0, \quad D_{0+}^{\beta} u(1)=\sum_{i=1}^{\infty} \eta_{i} D_{0+}^{\beta} u\left(\xi_{i}\right),
\end{array}\right.
$$

where $\alpha \geq 2, n-1<\alpha \leq n, 0<\beta<\alpha-1, \eta_{i} \in \mathbb{R}, 0<\xi_{1}<\cdots<\xi_{i}<\xi_{i+1}<\cdots<1(i=1,2, \ldots)$, $\sum_{i=1}^{\infty} \eta_{i} \xi_{i}^{\alpha-\beta-1} \neq 1, D_{0+}^{\alpha}$ is the standard Riemann-Liouville derivative, $f:[0,1] \times[0,+\infty) \rightarrow$ $[0,+\infty)$ is continuous, $a(t) \in C((0,1),[0,+\infty))$ may be singular at $t=0,1$.

FDE serve as an excellent instrument for the description of memory and hereditary properties of various materials and processes. During the last few decades, much attention has been paid to the study of boundary value problems (BVP for short) of fractional differential equation, such as the nonlocal BVP $[1,3,7,13,18]$, singular BVP $[6,8,11$, $19,20,25]$, semipositone BVP [14-16, 23], resonant BVP [2, 12], and impulsive BVP [10, 27]. Since only positive solutions are meaningful in most practical problems, some work has been done to study the existence of positive solutions for fractional boundary value problems by using the techniques of nonlinear analysis. In [16], the authors discussed the following fractional $\mathrm{m}$-point BVP:

$$
\left\{\begin{array}{l}
D_{0+}^{\alpha} u(t)+f(t, u(t))=0, \quad 0<t<1,1<\alpha \leq 2, \\
u(0)=0, \quad D_{0+}^{\beta} u(1)=\sum_{i=1}^{m-2} \eta_{i} D_{0+}^{\beta} u\left(\xi_{i}\right)
\end{array}\right.
$$

where $0<\beta<\alpha-1,0<\xi_{1}<\cdots<\xi_{m-2}<1$ with $\sum_{i=1}^{m-2} \eta_{i} \xi_{i}^{\alpha-\beta-1}<1$. Some new positive properties of the corresponding Green function were established, and a number of theo-

(c) The Author(s) 2018. This article is distributed under the terms of the Creative Commons Attribution 4.0 International License (http://creativecommons.org/licenses/by/4.0/), which permits unrestricted use, distribution, and reproduction in any medium, provided you give appropriate credit to the original author(s) and the source, provide a link to the Creative Commons license, and indicate if changes were made. 
rems on the existence of positive solutions were obtained. However, there are few works on the uniqueness of solution for fractional boundary value problems $[4,5,9,17,21,22$, $24,26]$. To obtain the uniqueness of solution, the main tool used in most of the works in literature is the Banach contraction map principle provided the nonlinearity satisfies the Lipschitz condition. Liang and Zhang [9] studied the uniqueness of positive solution for the following fractional three-point BVP:

$$
\left\{\begin{array}{l}
D_{0+}^{\alpha} u(t)+f(t, u(t))=0, \quad 0<t<1,3<\alpha \leq 4, \\
u(0)=u^{\prime}(0)=u^{\prime \prime}(0)=0, \quad u^{\prime \prime}(1)=\beta u^{\prime \prime}(\eta),
\end{array}\right.
$$

where $0<\eta<1$ with $0<\beta \eta^{\alpha-3}<1, f:[0,1] \times[0,+\infty) \rightarrow[0,+\infty)$ is continuous and nondecreasing with respect to the second variable, and there exists

$$
0<\lambda<\left(\frac{2}{(\alpha-2) \Gamma(\alpha+1)}+\frac{\beta \eta^{\alpha-3}(1-\eta)}{(\alpha-2)\left(1-\beta \eta^{\alpha-3}\right) \Gamma(\alpha)}\right)^{-1}
$$

such that, for $u, v \in[0,+\infty)$ with $u \geq v$ and $t \in[0,1]$,

$$
f(t, u)-f(t, v) \leq \lambda \ln (1+u-v) .
$$

It should be noted that (1.2) implies $f(t, u)-f(t, v) \leq \lambda(u-v)$ since $\ln (1+x) \leq x, \forall x \geq 0$, that is, $f$ satisfies the Lipschitz condition.

Motivated by the above works, in this paper we aim to establish the existence of uniqueness and the nonexistence of positive solution to BVP (1.1). It is well known that the cone plays a very important role in seeking positive solution. Our analysis relies on the iterative technique on the cone derived from the properties of the Green function. Our work presented in this paper has the following features. Firstly, the uniqueness results are obtained under some conditions concerning the spectral radius with respect to the relevant linear operator. Secondly, the Lipschitz condition is generalized. In addition, the error estimation of iterative sequences is also given. Thirdly, the nonexistence results of positive solution are obtained under conditions concerning the spectral radius of the relevant linear operator. Finally, we impose weaker positivity conditions on the nonlocal boundary term, that is, some of the coefficients $\eta_{i}$ can be negative.

\section{Preliminaries and lemmas}

In this section, we present some notations and lemmas that will be used in the proof of our main results.

Definition 2.1 The fractional integral of order $\alpha>0$ of a function $u:(0,+\infty) \rightarrow R$ is given by

$$
I_{0+}^{\alpha} u(t)=\frac{1}{\Gamma(\alpha)} \int_{0}^{t}(t-s)^{\alpha-1} u(s) d s
$$

provided that the right-hand side is point-wise defined on $(0,+\infty)$. 
Definition 2.2 The Riemann-Liouville fractional derivative of order $\alpha>0$ of a function $u:(0,+\infty) \rightarrow R$ is given by

$$
D_{0+}^{\alpha} u(t)=\frac{1}{\Gamma(n-\alpha)}\left(\frac{d}{d t}\right)^{n} \int_{0}^{t}(t-s)^{n-\alpha-1} u(s) d s
$$

where $n=[\alpha]+1,[\alpha]$ denotes the integer part of number $\alpha$, provided that the right-hand side is point-wise defined on $(0,+\infty)$.

Set

$$
\begin{aligned}
& K(t, s)=\frac{1}{\Gamma(\alpha)} \begin{cases}t^{\alpha-1}(1-s)^{\alpha-\beta-1}, & 0 \leq t \leq s \leq 1, \\
t^{\alpha-1}(1-s)^{\alpha-\beta-1}-(t-s)^{\alpha-1}, & 0 \leq s \leq t \leq 1,\end{cases} \\
& p(s)=\sum_{s \leq \xi_{i}} \eta_{i}\left(\xi_{i}-s\right)^{\alpha-\beta-1}, \\
& G(t, s)=K(t, s)+q(s) t^{\alpha-1},
\end{aligned}
$$

where

$$
q(s)=\frac{p(0)(1-s)^{\alpha-\beta-1}-p(s)}{\Gamma(\alpha)[1-p(0)]}, \quad p(0)=\sum_{i=1}^{\infty} \eta_{i} \xi_{i}^{\alpha-\beta-1} .
$$

Remark 2.1 Assume that $\sum_{i=1}^{\infty} \eta_{i} \xi_{i}^{\alpha-\beta-1}$ is convergent. Then $p \in C[0,1]$.

Remark 2.2 If $\eta_{i}=0(i=1,2, \ldots)$, we have $p(s) \equiv 0$ and $q(s) \equiv 0$; if $\eta_{i} \geq 0(i=1,2, \ldots)$ and $p(0)<1$, we have $q(s) \geq 0$ on $[0,1]$.

For the convenience in presentation, we here list the assumption to be used throughout the paper.

$\left(A_{1}\right) p(0) \neq 1, q(s) \geq 0$ on $[0,1]$

$\left(A_{2}\right) f:[0,1] \times[0,+\infty) \rightarrow[0,+\infty)$ is continuous, $a(t) \in C((0,1),[0,+\infty))$, and

$$
0<\int_{0}^{1} a(s) d s<+\infty .
$$

Lemma 2.1 Assume that $y \in L[0,1]$ and $p(0) \neq 1$. Then the unique solution of the problem

$$
\left\{\begin{array}{l}
D_{0+}^{\alpha} u(t)+y(t)=0, \quad 0<t<1, \\
u(0)=u^{\prime}(0)=\cdots=u^{(n-2)}(0)=0, \quad D_{0+}^{\beta} u(1)=\sum_{i=1}^{\infty} \eta_{i} D_{0+}^{\beta} u\left(\xi_{i}\right),
\end{array}\right.
$$

is

$$
u(t)=\int_{0}^{1} G(t, s) y(s) d s
$$

Proof The proof is similar to Lemma 2.3 in [17], so we omit it.

Lemma 2.2 The function $K(t, s)$ has the following properties: 
(1) $K(t, s)>0, \forall t, s \in(0,1)$;

(2) $K(t, s) \leq \frac{t^{\alpha-1}(1-s)^{\alpha-\beta-1}}{\Gamma(\alpha)}, \forall t, s \in[0,1]$;

(3) $\frac{t^{\alpha-1}}{\Gamma(\alpha)} h(s) \leq K(t, s) \leq \frac{M_{1}}{\Gamma(\alpha)} h(s), \forall t, s \in[0,1]$, where

$$
h(s)=\left[1-(1-s)^{\beta}\right](1-s)^{\alpha-\beta-1}, \quad M_{1}=\max \left\{1, \beta^{-1}\right\} .
$$

Proof It is obvious that (1), (2) hold. In the following, we will prove (3).

Case (i): $0<s \leq t<1$.

If $0<\beta<1$, we have

$$
\frac{\partial}{\partial t}\left[t^{\alpha-1}-\frac{(t-s)^{\alpha-1}}{(1-s)^{\alpha-2}}\right]=(\alpha-1) t^{\alpha-2}\left[1-\left(\frac{t-s}{t(1-s)}\right)^{\alpha-2}\right] \geq 0
$$

It follows from

$$
\frac{\partial}{\partial s}\left[\beta s+(1-s)^{\beta}\right] \leq 0, \quad \forall s \in[0,1)
$$

that

$$
s \leq \beta^{-1}\left[1-(1-s)^{\beta}\right], \quad \forall s \in[0,1] .
$$

It follows from (2.1) and (2.2) that

$$
\begin{aligned}
t^{\alpha-1}(1-s)^{\alpha-\beta-1}-(t-s)^{\alpha-1} & =(1-s)^{\alpha-\beta-1}\left[t^{\alpha-1}-\frac{(t-s)^{\alpha-1}}{(1-s)^{\alpha-\beta-1}}\right] \\
& \leq(1-s)^{\alpha-\beta-1}\left[t^{\alpha-1}-\frac{(t-s)^{\alpha-1}}{(1-s)^{\alpha-2}}\right] \\
& \leq s(1-s)^{\alpha-\beta-1} \\
& \leq \beta^{-1} h(s) .
\end{aligned}
$$

If $\beta \geq 1$, we have

$$
\begin{aligned}
& \frac{\partial}{\partial t}\left[t^{\alpha-1}(1-s)^{\alpha-\beta-1}-(t-s)^{\alpha-1}\right] \\
& \quad=(\alpha-1)\left[t^{\alpha-2}(1-s)^{\alpha-\beta-1}-(t-s)^{\alpha-2}\right] \\
& \quad \geq(\alpha-1)\left[(t-t s)^{\alpha-2}-(t-s)^{\alpha-2}\right] \geq 0 .
\end{aligned}
$$

Therefore,

$$
t^{\alpha-1}(1-s)^{\alpha-\beta-1}-(t-s)^{\alpha-1} \leq(1-s)^{\alpha-\beta-1}\left[1-(1-s)^{\beta}\right]=h(s) .
$$

On the other hand, noticing $0<\beta<\alpha-1$, we have

$$
\begin{aligned}
& t^{\alpha-1}(1-s)^{\alpha-\beta-1}-(t-s)^{\alpha-1} \\
& \quad \geq t^{\alpha-1}(1-s)^{\alpha-\beta-1}-(t-s)^{\beta}(t-t s)^{\alpha-\beta-1}
\end{aligned}
$$




$$
\begin{aligned}
& =\left[1-\left(1-\frac{s}{t}\right)^{\beta}\right] t^{\alpha-1}(1-s)^{\alpha-\beta-1} \\
& \geq\left[1-(1-s)^{\beta}\right] t^{\alpha-1}(1-s)^{\alpha-\beta-1} \\
& =t^{\alpha-1} h(s) .
\end{aligned}
$$

Case (ii): $0 \leq t \leq s \leq 1$.

If $\beta \geq 1$, we have

$$
\begin{aligned}
t^{\alpha-1}(1-s)^{\alpha-\beta-1} & \leq s^{\alpha-1}(1-s)^{\alpha-\beta-1} \\
& \leq\left[1-(1-s)^{\beta}\right](1-s)^{\alpha-\beta-1}=h(s) .
\end{aligned}
$$

If $0<\beta<1$. It follows from (2.2) that

$$
t^{\alpha-1}(1-s)^{\alpha-\beta-1} \leq s^{\alpha-1}(1-s)^{\alpha-\beta-1} \leq s(1-s)^{\alpha-\beta-1} \leq \beta^{-1} h(s) .
$$

On the other hand, noticing $0<\beta<\alpha-1$, we have

$$
t^{\alpha-1}(1-s)^{\alpha-\beta-1} \geq\left[1-(1-s)^{\beta}\right] t^{\alpha-1}(1-s)^{\alpha-\beta-1}=t^{\alpha-1} h(s) .
$$

It follows from (2.3)-(2.8) that (3) holds.

Lemma 2.3 The function $G(t, s)$ has the following properties:

(1) $G(t, s)>0, \forall t, s \in(0,1)$;

(2) $G(t, s) \leq t^{\alpha-1} \Phi_{1}(s), \forall t, s \in[0,1]$;

(3) $t^{\alpha-1} \Phi_{2}(s) \leq G(t, s) \leq M_{1} \Phi_{2}(s), \forall t, s \in[0,1]$,

where

$$
\Phi_{1}(s)=\frac{(1-s)^{\alpha-\beta-1}}{\Gamma(\alpha)}+q(s), \quad \Phi_{2}(s)=\frac{h(s)}{\Gamma(\alpha)}+q(s) .
$$

Proof It can be directly deduced from Lemma 2.2 and the definition of $G(t, s)$, so we omit the proof.

Let $E=C[0,1]$ be endowed with the maximum norm $\|u\|=\max _{0 \leq t \leq 1}|u(t)|, B_{r}=\{u \in E$ : $\|u\|<r\}$. Define cones $P, Q$ by

$$
\begin{aligned}
& P=\{u \in E: u(t) \geq 0\}, \\
& Q=\left\{u \in E: u(t) \geq M_{1}^{-1} t^{\alpha-1}\|u\|\right\} .
\end{aligned}
$$

Lemma 2.4 Define $A: P \rightarrow E$ as follows:

$$
A u(t)=\int_{0}^{1} G(t, s) a(s) f(s, u(s)) d s
$$

then $A: P \rightarrow Q$ is completely continuous. 
Proof By Lemma 2.3, it is easy to check that $A: P \rightarrow Q$. By means of the Arzela-Ascoli theorem, $A: P \rightarrow Q$ is completely continuous.

Remark 2.3 Lemma 2.4 is valid if $\left(A_{2}\right)$ is replaced by $\left(A_{2}^{\prime}\right) f:[0,1] \times[0,+\infty) \rightarrow[0,+\infty)$ is continuous, $a(t) \in C((0,1),[0,+\infty))$ and

$$
0<\int_{0}^{1} a(s) \Phi_{i}(s) d s<+\infty, \quad i=1,2 .
$$

Let

$$
T u(t)=\int_{0}^{1} G(t, s) a(s) u(s) d s
$$

It is clear that $T$ is a completely continuous linear operator and $T(P) \subset Q$. By virtue of the Krein-Rutmann theorem and Lemma 2.3, we have the following lemma.

Lemma 2.5 The spectral radius $r(T)>0$ and $T$ has a positive eigenfunction $\varphi_{1}$ corresponding to its first eigenvalue $(r(T))^{-1}$, that is, $T \varphi_{1}=r(T) \varphi_{1}$.

\section{Main results}

\subsection{Uniqueness results}

Theorem 3.1 Suppose that $f(t, 0) \not \equiv 0$ on $[0,1]$, and there exists $\lambda \in C(0,1) \cap L[0,1]$ satisfying

$$
0<\int_{0}^{1} a(s) \lambda(s) d s<+\infty
$$

such that

$$
|f(t, x)-f(t, y)| \leq \lambda(t)|x-y|, \quad t \in[0,1], x, y \in[0, \infty)
$$

Then (1.1) has a unique positive solution if the spectral radius $r\left(L_{\lambda}\right) \in(0,1)$, where

$$
L_{\lambda} u(t)=\int_{0}^{1} G(t, s) a(s) \lambda(s) u(s) d s
$$

Proof It follows from $f(t, 0) \not \equiv 0$ that $\theta$ is not a fixed point of $A$. Then we only need to prove that $A$ has a unique fixed point in $Q$.

Firstly, we will prove that $A$ has a fixed point in $Q$.

Set

$$
Q_{1}=\left\{u \in P: \exists l_{1}, l_{2}>0 \text { such that } l_{2} t^{\alpha-1} \leq u(t) \leq l_{1} t^{\alpha-1}\right\}
$$

For any $u \in Q \backslash\{\theta\}$, let

$$
l_{i}(u)=\int_{0}^{1} \Phi_{i}(s) a(s) \lambda(s) u(s) d s, \quad i=1,2 .
$$


It follows from Lemma 2.3 that $l_{1}(u), l_{2}(u)>0$ and

$$
l_{2}(u) t^{\alpha-1} \leq\left(L_{\lambda} u\right)(t) \leq l_{1}(u) t^{\alpha-1} .
$$

Therefore,

$$
L_{\lambda}: Q \backslash\{\theta\} \rightarrow Q_{1} .
$$

Similar to Lemma 2.5, we have that the spectral radius $r\left(L_{\lambda}\right)>0$ and $L_{\lambda}$ has a positive eigenfunction $\psi_{1}$, that is, $L_{\lambda} \psi_{1}=r\left(L_{\lambda}\right) \psi_{1}$. It is easy to see that

$$
\left(r\left(L_{\lambda}\right)\right)^{-1} l_{2}\left(\psi_{1}\right) t^{\alpha-1} \leq \psi_{1}(t) \leq\left(r\left(L_{\lambda}\right)\right)^{-1} l_{1}\left(\psi_{1}\right) t^{\alpha-1} .
$$

For any $u_{0} \in Q \backslash\{\theta\}$, let

$$
u_{n}=A\left(u_{n-1}\right), \quad n=1,2, \ldots
$$

We may suppose that $u_{1}-u_{0} \neq \theta$ (otherwise, the proof is finished). It follows from (3.1) and (3.2) that

$$
L_{\lambda}\left(\left|u_{1}-u_{0}\right|\right) \leq \frac{r\left(L_{\lambda}\right) l_{1}\left(\left|u_{1}-u_{0}\right|\right)}{l_{2}\left(\psi_{1}\right)} \psi_{1} .
$$

Then

$$
\begin{aligned}
&\left|u_{2}-u_{1}\right|=\left|\int_{0}^{1} G(t, s) a(s)\left[f\left(s, u_{1}(s)\right)-f\left(s, u_{0}(s)\right)\right] d s\right| \\
& \leq \int_{0}^{1} G(t, s) a(s) \lambda(s)\left|u_{1}(s)-u_{0}(s)\right| d s \\
&=L_{\lambda}\left(\left|u_{1}-u_{0}\right|\right) \\
& \leq \frac{r\left(L_{\lambda}\right) l_{1}\left(\left|u_{1}-u_{0}\right|\right)}{l_{2}\left(\psi_{1}\right)} \psi_{1}, \\
&\left|u_{3}-u_{2}\right|=\left|\int_{0}^{1} G(t, s) a(s)\left[f\left(s, u_{2}(s)\right)-f\left(s, u_{1}(s)\right)\right] d s\right| \\
& \leq \int_{0}^{1} G(t, s) a(s) \lambda(s)\left|u_{2}(s)-u_{1}(s)\right| d s \\
&=L_{\lambda}\left(\left|u_{2}-u_{1}\right|\right) \\
& \leq \frac{r\left(L_{\lambda}\right) l_{1}\left(\left|u_{1}-u_{0}\right|\right)}{l_{2}\left(\psi_{1}\right)} L_{\lambda} \psi_{1} \\
&=\frac{\left[r\left(L_{\lambda}\right)\right]^{2} l_{1}\left(\left|u_{1}-u_{0}\right|\right)}{l_{2}\left(\psi_{1}\right)} \psi_{1}, \\
& \ldots \\
&\left|u_{m+1}-u_{m}\right|=\left|\int_{0}^{1} G(t, s) a(s)\left[f\left(s, u_{m}(s)\right)-f\left(s, u_{m-1}(s)\right)\right] d s\right|
\end{aligned}
$$




$$
\begin{aligned}
& \leq \int_{0}^{1} G(t, s) a(s) \lambda(s)\left|u_{m}(s)-u_{m-1}(s)\right| d s \\
& =L_{\lambda}\left(\left|u_{m}-u_{m-1}\right|\right) \\
& \leq \frac{\left[r\left(L_{\lambda}\right)\right]^{m-1} l_{1}\left(\left|u_{1}-u_{0}\right|\right)}{l_{2}\left(\psi_{1}\right)} L_{\lambda} \psi_{1} \\
& =\frac{\left[r\left(L_{\lambda}\right)\right]^{m} l_{1}\left(\left|u_{1}-u_{0}\right|\right)}{l_{2}\left(\psi_{1}\right)} \psi_{1} .
\end{aligned}
$$

By induction, we can get

$$
\left|u_{n+1}-u_{n}\right| \leq \frac{\left[r\left(L_{\lambda}\right)\right]^{n} l_{1}\left(\left|u_{1}-u_{0}\right|\right)}{l_{2}\left(\psi_{1}\right)} \psi_{1}, \quad n=1,2, \ldots
$$

Then, for any $n, m \in \mathbb{N}$, we have

$$
\begin{aligned}
\left|u_{n+m}-u_{n}\right| & \leq\left|u_{n+m}-u_{n+m-1}\right|+\cdots+\left|u_{n+1}-u_{n}\right| \\
& \leq\left(\left[r\left(L_{\lambda}\right)\right]^{n+m-1}+\cdots+\left[r\left(L_{\lambda}\right)\right]^{n}\right) \frac{l_{1}\left(\left|u_{1}-u_{0}\right|\right)}{l_{2}\left(\psi_{1}\right)} \psi_{1} \\
& \leq \frac{\left[r\left(L_{\lambda}\right)\right]^{n} l_{1}\left(\left|u_{1}-u_{0}\right|\right)}{\left[1-r\left(L_{\lambda}\right)\right] l_{2}\left(\psi_{1}\right)} \psi_{1} .
\end{aligned}
$$

It follows from $r\left(L_{\lambda}\right)<1$ that

$$
\left\|u_{n+m}-u_{m}\right\| \rightarrow 0 \quad(n \rightarrow \infty),
$$

which implies $\left\{u_{n}\right\}$ is a Cauchy sequence. Therefore, there exists $u^{*} \in Q$ such that $\left\{u_{n}\right\}$ converges to $u^{*}$. Clearly $u^{*}$ is a fixed point of $A$.

In the following, we will prove that the fixed point of $A$ is unique.

Suppose that $v \neq u^{*}$ is a positive fixed point of $A$. Then there exists $l_{1}\left(\left|u^{*}-v\right|\right)>0$ such that

$$
L_{\lambda}\left(\left|u^{*}-v\right|\right) \leq \frac{r\left(L_{\lambda}\right) l_{1}\left(\left|u^{*}-v\right|\right)}{l_{2}\left(\psi_{1}\right)} \psi_{1} .
$$

Therefore,

$$
\begin{aligned}
\left|A u^{*}-A v\right| & =\left|\int_{0}^{1} G(t, s) a(s)\left[f\left(s, u^{*}(s)\right)-f(s, v(s))\right] d s\right| \\
& \leq \int_{0}^{1} G(t, s) a(s) \lambda(s)\left|u^{*}(s)-v(s)\right| d s \\
& \leq \frac{r\left(L_{\lambda}\right) l_{1}\left(\left|u^{*}-v\right|\right)}{l_{2}\left(\psi_{1}\right)} \psi_{1} .
\end{aligned}
$$

Similar to the proof of (3.7), we can get

$$
\left|A^{n} u^{*}-A^{n} v\right| \leq \frac{\left[r\left(L_{\lambda}\right)\right]^{n} l_{1}\left(\left|u^{*}-v\right|\right)}{l_{2}\left(\psi_{1}\right)} \psi_{1} .
$$


It follows from $r\left(L_{\lambda}\right)<1$ that

$$
\left\|u^{*}-v\right\|=\left\|A^{n} u^{*}-A^{n} v\right\| \rightarrow 0 \quad(n \rightarrow \infty)
$$

which implies that the positive fixed point of $A$ is unique.

Remark 3.1 The unique positive solution $u^{*}$ of (1.1) can be approximated by the iterative schemes: for any $u_{0} \in Q \backslash\{\theta\}$, let

$$
u_{n}=A\left(u_{n-1}\right), \quad n=1,2, \ldots
$$

then $u_{n} \rightarrow u^{*}$. Furthermore, we have error estimation

$$
\left|u_{n}-u^{*}\right| \leq \frac{\left[r\left(L_{\lambda}\right)\right]^{n} l_{1}\left(\left|u_{1}-u_{0}\right|\right)}{\left[1-r\left(L_{\lambda}\right)\right] l_{2}\left(\psi_{1}\right)} \psi_{1}
$$

and with the rate of convergence

$$
\left\|u_{n}-u^{*}\right\|=O\left(\left[r\left(L_{\lambda}\right)\right]^{n}\right) .
$$

Remark 3.2 The spectral radius satisfies $r\left(L_{\lambda}\right)=\lim _{n \rightarrow \infty}\left\|L_{\lambda}^{n}\right\|^{\frac{1}{n}}$ and $r\left(L_{\lambda}\right) \leq\left\|L_{\lambda}^{n}\right\|^{\frac{1}{n}}$. Particularly,

$$
r\left(L_{\lambda}\right) \leq\left\|L_{\lambda}\right\|=\sup _{0 \leq t \leq 1} \int_{0}^{1} G(t, s) a(s) \lambda(s) d s .
$$

\subsection{Nonexistence results}

Theorem 3.2 Suppose that there exists $b_{1} \in C(0,1) \cap L[0,1]$ satisfying

$$
0<\int_{0}^{1} a(s) b_{1}(s) d s<+\infty
$$

such that

$$
f(t, x) \leq b_{1}(t) x, \quad t \in[0,1], x \in[0, \infty) .
$$

Then (1.1) has no positive solution if the spectral radius $r\left(L_{b_{1}}\right) \in(0,1)$, where

$$
L_{b_{1}} u(t)=\int_{0}^{1} G(t, s) a(s) b_{1}(s) u(s) d s
$$

Proof We only need to prove that $A$ has no fixed point in $Q_{1}$. If otherwise, there exists $v \in Q_{1}$ such that $A v=v$. Similar to Lemma 2.5, we have that the spectral radius $r\left(L_{b_{1}}\right)>0$ and $L_{b_{1}}$ has a positive eigenfunction $\varphi_{b_{1}}$ satisfying

$$
L_{b_{1}} \varphi_{b_{1}}=r\left(L_{b_{1}}\right) \varphi_{b_{1}} .
$$

It is clear that $v, \varphi_{b_{1}} \in Q_{1}$. Therefore, there exists $c_{1}>0$ such that

$$
v \leq c_{1} \varphi_{b_{1}}
$$


By $f(t, x) \leq b_{1}(t) x$, we have $v=A v \leq L_{b_{1}} v$. It is obvious that $L_{b_{1}}$ is increasing in $Q_{1}$. By induction, we can get $v \leq L_{b_{1}}^{n} v, \forall n=1,2,3, \ldots$. Then

$$
v \leq L_{b_{1}}^{n} \nu \leq L_{b_{1}}^{n} c_{1} \varphi_{b_{1}}=c_{1}\left[r\left(L_{b_{1}}\right)\right]^{n} \varphi_{b_{1}}, \quad \forall n=1,2,3, \ldots
$$

Noticing $r\left(L_{b_{1}}\right)<1$, we have $v=\theta$, which contradicts $v \in Q_{1}$.

Theorem 3.3 Suppose that there exists $b_{2} \in C(0,1) \cap L[0,1]$ satisfying

$$
0<\int_{0}^{1} a(s) b_{2}(s) d s<+\infty
$$

such that

$$
f(t, x) \geq b_{2}(t) x, \quad t \in[0,1], x \in[0, \infty) .
$$

Then (1.1) has no positive solution if the spectral radius $r\left(L_{b_{2}}\right)>1$, where

$$
L_{b_{2}} u(t)=\int_{0}^{1} G(t, s) a(s) b_{2}(s) u(s) d s
$$

Proof The proof is just similar to Theorem 3.2, so we omit it.

\section{Example}

Example 4.1 Consider the following problem:

$$
\left\{\begin{array}{l}
D_{0+}^{\frac{5}{2}} u(t)+a(t) f(t, u(t))=0, \quad 0<t<1, \\
u(0)=u^{\prime}(0)=0, \quad D_{0+}^{\frac{1}{2}} u(1)=\sum_{i=1}^{\infty} \eta_{i} D_{0+}^{\frac{1}{2}} u\left(\xi_{i}\right),
\end{array}\right.
$$

where

$$
\begin{aligned}
& \eta_{i}=\frac{1}{2^{4+i}}, \quad \xi_{i}=1-\frac{1}{2^{i}}, \quad i=1,2, \ldots, \\
& a(t)=\sqrt{\frac{100 \pi}{3 t}}, \quad f(t, x)=1+\sqrt{t}|\sin x| .
\end{aligned}
$$

Let $\lambda(t)=\sqrt{t}$, we have

$$
|f(t, x)-f(t, y)| \leq \lambda(t)|x-y|, \quad t \in[0,1], x, y \in[0, \infty) .
$$

It is clear that

$$
\begin{aligned}
& K(t, s)=\frac{1}{\Gamma\left(\frac{5}{2}\right)} \begin{cases}t^{\frac{3}{2}}(1-s), & 0 \leq t \leq s \leq 1, \\
t^{\frac{3}{2}}(1-s)-(t-s)^{\frac{3}{2}}, & 0 \leq s \leq t \leq 1,\end{cases} \\
& p(0)=\sum_{i=1}^{\infty} \eta_{i} \xi_{i}=\frac{1}{24},
\end{aligned}
$$




$$
\begin{aligned}
& p(s)= \begin{cases}\sum_{i=1}^{\infty} \eta_{i}\left(\xi_{i}-s\right), & s \in\left[0, \xi_{1}\right], \\
\sum_{i=2}^{\infty} \eta_{i}\left(\xi_{i}-s\right), s \in\left(\xi_{1}, \xi_{2}\right], & \\
\cdots & s \in\left(\xi_{n}, \xi_{n+1}\right], \\
\sum_{i=n+1}^{\infty} \eta_{i}\left(\xi_{i}-s\right), & \\
\cdots, & \end{cases} \\
& q(s)=\frac{p(0)(1-s)-p(s)}{\Delta}, \\
& G(t, s)=K(t, s)+q(s) t^{\frac{3}{2}},
\end{aligned}
$$

where $\Delta:=\Gamma\left(\frac{5}{2}\right)[1-p(0)]$.

Let

$$
\begin{aligned}
& h_{1}(t)=\int_{0}^{1} K(t, s) d s \\
& h_{2}(t)=\int_{0}^{1} K(t, s) \int_{0}^{1} K(s, \tau) d \tau d s .
\end{aligned}
$$

Then

$$
\begin{aligned}
& h_{1}(t)=\frac{1}{\Gamma\left(\frac{5}{2}\right)}\left(\frac{t^{\frac{3}{2}}}{2}-\frac{2 t^{\frac{5}{2}}}{5}\right) \\
& h_{2}(t)=\int_{0}^{1} K(t, s) h_{1}(s) d s=\frac{32 t^{\frac{3}{2}}}{567 \pi}-\frac{t^{4}}{48}+\frac{t^{5}}{120} .
\end{aligned}
$$

By direct calculations, we have

$$
\begin{aligned}
& \max _{0 \leq t \leq 1} h_{1}(t)=h_{1}\left(\frac{3}{4}\right)=\frac{\sqrt{3}}{10 \sqrt{\pi}}, \\
& \max _{0 \leq t \leq 1} h_{2}(t) \approx h_{2}(0.7744) \approx 0.0070708134,
\end{aligned}
$$

and

$$
\begin{aligned}
\int_{0}^{1} q(s) d s & =\frac{p(0)}{2 \Delta}-\frac{1}{\Delta} \sum_{i=1}^{\infty} \frac{\eta_{i} \xi_{i}^{2}}{2} \\
& =\frac{1}{2 \Delta} \sum_{i=1}^{\infty} \eta_{i} \xi_{i}\left(1-\xi_{i}\right) \\
& \approx 0.00467238 .
\end{aligned}
$$

It is clear that

$$
\begin{aligned}
\left\|L_{\lambda}\right\| & =\sup _{0 \leq t \leq 1} \int_{0}^{1} \sqrt{\frac{100 \pi}{3}} G(t, s) d s \\
& \geq \int_{0}^{1} \sqrt{\frac{100 \pi}{3}} G\left(\frac{3}{4}, s\right) d s
\end{aligned}
$$




$$
\begin{aligned}
& =\sqrt{\frac{100 \pi}{3}} h_{1}\left(\frac{3}{4}\right)+\sqrt{\frac{100 \pi}{3}}\left(\frac{3}{4}\right)^{\frac{3}{2}} \int_{0}^{1} q(s) d s \\
& >\sqrt{\frac{100 \pi}{3}} h_{1}\left(\frac{3}{4}\right)=1 .
\end{aligned}
$$

On the other hand, we have

$$
\left\|L_{\lambda}^{2}\right\|=\sup _{0 \leq t \leq 1} \int_{0}^{1} \sqrt{\frac{100 \pi}{3}} G(t, s) \int_{0}^{1} \sqrt{\frac{100 \pi}{3}} G(s, \tau) d \tau d s .
$$

Noticing that

$$
G(t, s)=K(t, s)+q(s) t^{\frac{3}{2}} \leq K(t, s)+q(s),
$$

we have

$$
\begin{aligned}
& \int_{0}^{1} \sqrt{\frac{100 \pi}{3}} G(t, s) \int_{0}^{1} \sqrt{\frac{100 \pi}{3}} G(s, \tau) d \tau d s \\
& \quad=\frac{100 \pi}{3} \int_{0}^{1} G(t, s) \int_{0}^{1} G(s, \tau) d \tau d s \\
& \quad \leq \frac{100 \pi}{3} \int_{0}^{1}[K(t, s)+q(s)] \int_{0}^{1}[K(s, \tau)+q(\tau)] d \tau d s \\
& \quad=\frac{100 \pi}{3}\left[h_{2}(t)+h_{1}(t) \int_{0}^{1} q(\tau) d \tau+\int_{0}^{1} q(s) h_{1}(s) d s+\left(\int_{0}^{1} q(s) d s\right)^{2}\right] \\
& \quad \leq \frac{100 \pi}{3}\left[\left\|h_{2}\right\|+2\left\|h_{1}\right\| \int_{0}^{1} q(s) d s+\left(\int_{0}^{1} q(s) d s\right)^{2}\right] \\
& \approx 0.83837
\end{aligned}
$$

which implies that

$$
\left\|L_{\lambda}^{2}\right\|^{\frac{1}{2}}<1
$$

It follows from Lemma 2.5 and Remark 3.2 that

$$
0<r\left(L_{\lambda}\right) \leq\left\|L_{\lambda}^{2}\right\|^{\frac{1}{2}}<1<\left\|L_{\lambda}\right\| .
$$

Therefore the assumptions of Theorem 3.1 are satisfied. Thus Theorem 3.1 ensures that BVP (4.1) has a unique positive solution.

\section{Conclusions}

In this paper, we consider both the existence of uniqueness and the nonexistence of positive solution for fractional differential equations with infinite-point boundary value conditions. The interesting point lies in that both the existence of uniqueness and the nonexistence of positive solution are closely associated with the spectral radius with respect to the relevant linear operator. 


\section{Acknowledgements}

The authors would like to thank the referees for their pertinent comments and valuable suggestions.

\section{Funding}

The authors are supported financially by the Natural Science Foundation of Shandong Province of China (ZR2017MA036, ZR2014AM034), the National Natural Science Foundation of China (11571296).

\section{Abbreviations}

FDE, Fractional differential equations; BVP, Boundary value problems.

\section{Availability of data and materials}

Data sharing not applicable to this article as no datasets were generated or analysed during the current study.

\section{Competing interests}

The authors declare that there is no conflict of interests regarding the publication of this paper.

\section{Authors' contributions}

All authors contributed equally to the writing of this paper. All authors read and approved the final manuscript.

\section{Author details}

'School of Statistics, Qufu Normal University, Qufu, P.R. China. ${ }^{2}$ School of Mathematical Sciences, Qufu Normal University, Qufu, P.R. China. ${ }^{3}$ Department of Mathematics and Statistics, Curtin University, Perth, Australia.

\section{Publisher's Note}

Springer Nature remains neutral with regard to jurisdictional claims in published maps and institutional affiliations.

Received: 21 March 2018 Accepted: 10 July 2018 Published online: 21 July 2018

\section{References}

1. Bai, Z:: On positive solutions of a nonlocal fractional boundary value problem. Nonlinear Anal. 72, $916-924$ (2010)

2. Bai, Z:: Solvability for a class of fractional m-point boundary value problem at resonance. Comput. Math. Appl. 62, 1292-1302 (2011)

3. Bai, Z., Zhang, Y.: Solvability of fractional three-point boundary value problems with nonlinear growth. Appl. Math. Comput. 218, 1719-1725 (2011)

4. Cui, Y.: Uniqueness of solution for boundary value problems for fractional differential equations. Appl. Math. Lett. 51 48-54 (2016)

5. Cui, Y., Ma, W., Sun, Q., Su, X.: New uniqueness results for boundary value problem of fractional differential equation. Nonlinear Anal., Model. Control 23, 31-39 (2018)

6. Guan, Y., Zhao, Z., Lin, X.: On the existence of positive solutions and negative solutions of singular fractional differential equations via global bifurcation techniques. Bound. Value Probl. 2016, Article ID 141 (2016)

7. Guo, L., Liu, L., Wu, Y.: Existence of positive solutions for singular higher-order fractional differential equations with infinite-points boundary conditions. Bound. Value Probl. 2016, Article ID 114 (2016)

8. Hao, X.: Positive solution for singular fractional differential equations involving derivatives. Adv. Differ. Equ. 2016 , Article ID 139 (2016)

9. Liang, S., Zhang, J.: Existence and uniqueness of strictly nondecreasing and positive solution for a fractional three-point boundary value problem. Comput. Math. Appl. 62, 1333-1340 (2011)

10. Wang, G., Ahmad, B., Zhang, L.: Existence results for nonlinear fractional differential equations with closed boundary conditions and impulses. Adv. Differ. Equ. 2012, Article ID 169 (2012)

11. Wang, Y., Liu, L.: Necessary and sufficient condition for the existence of positive solution to singular fractional differential equations. Adv. Differ. Equ. 2015, Article ID 207 (2015)

12. Wang, Y., Liu, L.: Positive solutions for a class of fractional 3-point boundary value problems at resonance. Adv. Differ Equ. 2017, Article ID 7 (2017)

13. Wang, Y., Liu, L., Wu, Y.: Positive solutions for a nonlocal fractional differential equation. Nonlinear Anal. 74, 3599-3605 (2011)

14. Wang, Y., Liu, L., Wu, Y.: Positive solutions for a class of fractional boundary value problem with changing sign nonlinearity. Nonlinear Anal. 74, 6434-6441 (2011)

15. Wang, Y., Liu, L., Wu, Y.: Positive solutions of a fractional boundary value problem with changing sign nonlinearity. Abstr. Appl. Anal. 2012, Article ID 149849 (2012)

16. Wang, Y., Liu, L., Wu, Y.: Positive solutions for a fractional boundary value problem with changing sign nonlinearity. Abstr. Appl. Anal. 2012, Article ID 214042 (2012)

17. Wang, Y., Liu, L., Wu, Y.: Existence and uniqueness of a positive solution to singular fractional differential equations. Bound. Value Probl. 2012, Article ID 81 (2012)

18. Zhai, C., Xu, L.: Properties of positive solutions to a class of four-point boundary value problem of Caputo fractional differential equations with a parameter. Commun. Nonlinear Sci. Numer. Simul. 19, 2820-2827 (2014)

19. Zhang, X., Liu, L., Wiwatanapataphee, B., Wu, Y.: The eigenvalue for a class of singular p-Laplacian fractional differential equations involving the Riemann-Stieltjes integral boundary condition. Appl. Math. Comput. 235, 412-422 (2014)

20. Zhang, X., Liu, L., Wu, Y.: Multiple positive solutions of a singular fractional differential equation with negatively perturbed term. Math. Comput. Model. 55, 1263-1274 (2012)

21. Zhang, X., Liu, L., Wu, Y.: The uniqueness of positive solution for a singular fractional differential system involving derivatives. Commun. Nonlinear Sci. Numer. Simul. 18, 1400-1409 (2013) 
22. Zhang, $X ., L i u, L ., W u, Y$ : The uniqueness of positive solution for a fractional order model of turbulent flow in a porous medium. Appl. Math. Lett. 37, 26-33 (2014)

23. Zhang, X., Shao, Z., Zhong, Q.: Positive solutions for semipositone $(k, n-k)$ conjugate boundary value problems with singularities on space variables. Appl. Math. Lett. 72, 50-57 (2017)

24. Zhang, X., Zhong, Q.: Uniqueness of solution for higher-order fractional differential equations with conjugate type integral conditions. Fract. Calc. Appl. Anal. 20, 1471-1484 (2017)

25. Zhang, X., Zhong, Q.: Triple positive solutions for nonlocal fractional differential equations with singularities both on time and space variables. Appl. Math. Lett. 80, 12-19 (2018)

26. Zou, Y., He, G.: On the uniqueness of solutions for a class of fractional differential equations. Appl. Math. Lett. 74, 68-73 (2017)

27. Zuo, M., Hao, X., Liu, L., Cui, Y.: Existence results for impulsive fractional integro-differential equation of mixed type with constant coefficient and antiperiodic boundary conditions. Bound. Value Probl. 2017, Article ID 161 (2017)

\section{Submit your manuscript to a SpringerOpen ${ }^{\circ}$ journal and benefit from:}

- Convenient online submission

- Rigorous peer review

- Open access: articles freely available online

High visibility within the field

- Retaining the copyright to your article

Submit your next manuscript at $\gg$ springeropen.com 